\title{
Ruxolitinib Suppresses Liver Fibrosis Progression and Accelerates Fibrosis Reversal via Selectively Targeting Janus Kinase 1/2
}

\section{Zhenghui Song}

Southern Medical University

Xinhui Liu

Southern Medical University

Wan Zhang

Southern Medical University

Yue Luo

Southern Medical University

Hua Xiao

Michigan State University

Yun Liu

XiangNan University

Guanqi Dai

Southern Medical University

Jian Hong

Jinan University

Aimin Li ( $\nabla$ liaimin2005@163.com )

Southern Medical University https://orcid.org/0000-0001-9459-5540

\section{Research Article}

Keywords: Janus Kinase 1, Janus Kinase 2, Liver Fibrosis, HSCs, Ruxolitinib

Posted Date: November 10th, 2021

DOI: https://doi.org/10.21203/rs.3.rs-1050686/v1

License: (c) (1) This work is licensed under a Creative Commons Attribution 4.0 International License. Read Full License

Version of Record: A version of this preprint was published at Journal of Translational Medicine on April 5th, 2022. See the published version at https://doi.org/10.1186/s12967-022-03366-y. 

Ruxolitinib suppresses liver fibrosis progression and accelerates fibrosis reversal via selectively targeting Janus kinase 1/2

Zhenghui Song ${ }^{1,2, \#, ~ X i n h u i ~ L i u 1, ~}{ }^{1,2,3, \#}$, Wan Zhang ${ }^{1,2}$, Yue Luo ${ }^{1,2,3}$, Hua Xiao ${ }^{3}$, Yun $\mathrm{Liu}^{4}$, Guanqi Dai ${ }^{1,2}$, Jian Hong ${ }^{5,{ }^{*}}$, Aimin $\mathrm{Li}^{1,2,3,{ }^{*}}$

${ }^{1}$ Department of Hepatology, Cancer Center, Integrated Hospital of Traditional Chinese Medicine, Southern Medical University, Guangzhou, 510315, China

2 School of Traditional Chinese Medicine, Southern Medical University, Guangzhou, Guangdong, 510515, China

${ }^{3}$ Department of Physiology, Michigan State University, East Lansing, MI, 48824, USA

${ }^{4}$ Department of Endocrinology and Metabolic Diseases, Affiliated Hospital (Clinical College) of Xiangnan University, Chenzhou, 423000, China

${ }^{5}$ School of Medicine, Jinan University, Guangzhou 510632, China

${ }^{*}$ Corresponding author

Email: liaimin2005@163.com (A. Li) and hongjian7@jnu.edu.cn (H. Jian)

Address: No.13 Shiliugang Road, Guangzhou, Guangdong, 510315, China.

\# These authors contributed equally to this work. 


\section{Abstract}

Background: JAK1 and JAK2 have been implicated in fibrosis and cancer as a fibroblast-related marker; however, their role in liver fibrosis has not been elucidated. Here, we aim to determine the effect and underlying mechanism of JAK1/2 inhibition on liver fibrosis and hepatic stellate cells (HSCs) and further explore the therapeutic efficacy of Ruxolitinib, a JAK1/2 selective inhibitor, on preventing and reversing liver fibrosis in mice.

Methods: Immunohistochemistry staining of JAK1 and JAK2 were performed on liver tissue in mice with hepatic fibrosis and human liver tissue microarray of liver cirrhosis and liver cancer. LX-2 cells treated with specific siRNA of JAK1 and JAK2 were used to analysis activation, proliferation and migration of HSCs regulated by JAK1/2. The effects of Ruxolitinib (JAK1/2 inhibitor) on liver fibrosis were studied in LX-2 cells and two progressive and reversible fibrosis animal models (carbon tetrachloride (CCl4), Thioacetamide (TAA)).

Results: We found that JAK1/2 expression was positively correlated with the progression of HCC in humans and the levels of liver fibrosis in mice. Silencing of JAK1/2 down-regulated their downstream signaling and inhibited proliferation, migration, and activation of HSCs in vitro, while Ruxolitinib had similar effects on HSCs. Importantly, Ruxolitinib significantly attenuated fibrosis progression, improved cell damage, and accelerated fibrosis reversal in the liver of mice treated with $\mathrm{CCl}_{4}$ or TAA.

Conclusions: JAK1/2 regulates the function of HSCs and plays an essential 
role in liver fibrosis and HCC development. Its inhibitor, Ruxolitinib, may be an effective drug for preventing and treating liver fibrosis.

\section{Keywords}

Janus Kinase 1; Janus Kinase 2; Liver Fibrosis; HSCs; Ruxolitinib

\section{Background}

Liver fibrosis is a chronic liver injury mainly caused by hepatitis virus B and C infection, alcohol abuse and nonalcoholic steatohepatitis (NASH) [1]. Liver fibrosis eventually progresses to liver cirrhosis, a common cause of death worldwide [2], resulting in serious complications, including portal hypertension, liver failure, and hepatocellular carcinoma (HCC), leading to failure of liver function and destruction of liver structures, and ultimately organ dysfunction and death $[3,4]$. Currently, liver transplantation is the only effective cure, which brings a tremendous economic burden to the patients [5]. Liver fibrosis is due to excessive accumulation of extracellular matrix (ECM) in response to chronic liver damage. Hepatic stellate cells (HSCs) are considered the most critical cell type for the production of collagens [6-8]. When stimulated by liver damage, HSCs will be activated, and then differentiate into myofibroblasts acquire fibrogenic property for producing ECM, resulting in fibrotic scar $[9,10]$. Many drug companies try to find methods to halting scarring or even remove existing scars, but no drug is yet approved for treating cirrhosis [11]. Thus, the 
development of effective anti-fibrotic drugs to stop progression to cirrhosis or even reverse advanced fibrosis is urgently needed [12].

Several signaling pathways are found to be related to liver fibrosis, and may be potential targets for treatment. The Janus kinase/Signal Transducer and Activator of Transcription protein (JAK/STAT) signaling pathway is a chain of interactions between proteins and involved in processes such as immunity, cell division, cell death, and tumor formation [13-19]. Recent studies have reported that STATs are associated with tissue fibrosis, including skin, lung, liver, systemic sclerosis (SSc), and STAT3 inhibitors have been shown to be effective in the Carbon tetrachloride $\left(\mathrm{CCl}_{4}\right)$-induced liver fibrosis model $[20,21]$. JAKs act as the upstream of STATs, and JAK/STAT signaling pathway plays a role in promoting the fibrosis, and selective JAK2 inhibitor can effectively block fibroblast activation improve fibrosis [22]. Besides, recent studies suggested that JAK1 might also play an indirect role in promoting fibrosis through the transphosphorylation of JAK2 [23]. However, the role of JAK1 and JAK2 in liver fibrosis remains unclear.

Ruxolitinib is the only effective small-molecule JAK1/2 selective inhibitor approved by the US Food and Drug Administration (FDA) for myelofibrosis treatment in 2011. It is mainly used for the treatment of intermediate or highrisk bone marrow fibers, which is well tolerated in a multicenter study and can effectively alleviate splenomegaly, improve spleen hyperfunction and systemic symptoms of patients [24-26]. Recently, it has been reported that Ruxolitinib is 
effective and safe for the treatment of hematological diseases such as polycythemia vera, lymphoma, etc.[27,28], and can benefit some patients with pancreatic cancer [28]. Currently, a series of clinical studies of Ruxolitinib in malignant glioma, multiple myeloma, lung cancer, breast cancer, colorectal cancer, head, and neck squamous cell carcinoma, and prostate cancer are underway (https://clinicaltrials.gov/ct2/results?cond=ruxolitinib). However, the efficacy of Ruxolitinib on liver fibrosis and liver cancer has not been explored. In the present study, we found up-regulated JAK1 and JAK2 were associated with liver disease in humans and mice, while knockdown of JAK1 and JAK2 inhibited activation, proliferation, and migration of HSCs. Also, Ruxolitinib, the JAK1/2 selective inhibitor, blocked HSCs activation, both in cell culture and in animal fibrosis models, included by $\mathrm{CCl}_{4}$ and Thioacetamide (TAA). Furthermore, we found that Ruxolitinib attenuated liver fibrosis progression, accelerated its reversal, and protected from liver damage, which provides a new direction and clinical transformation basis for the treatment of liver fibrosis and even early intervention of HCC.

\section{Methods}

\section{Cell culture}

Immortalized human HSC cell line (LX-2) was purchased from Chinese Academy of Sciences Cell Bank (Shanghai, China) and cultured in Dulbecco's modified Eagle's medium (DMEM) (Gibco, China), supplemented with $10 \%$ fetal 
bovine serum. (FBS) (Gibco, USA) and 1\% Penicillin/Streptomycin at $5 \% \mathrm{CO}_{2}$ and $37^{\circ} \mathrm{C}$. All cell experiments were performed in strict accordance with cell culture protocols.

\section{Chemicals}

For in vitro experiments, Ruxolitinib (INCB018424, Selleck chemicals, USA) was dissolved in DMSO and further diluted to the required concentration. For in vivo experiments, Ruxolitinib suspension was prepared in $0.5 \%$ carboxymethyl cellulose sodium normal saline solution.

\section{Small interfering RNA (siRNA) Transfection}

siRNA for JAK1 and JAK2 were designed and synthesized by RiboBio (Guangzhou, China). siRNAs were transfected into cells using Lipofectamine 3000 (Invitrogen) according to the manufacturer's protocol. Cells were collected after $48-72 \mathrm{~h}$ for further experiments.

\section{Human samples}

Human liver tissue microarray was purchased from Biomax, including 5 liver samples with normal liver tissue, 22 liver samples with cirrhosis, and 53 liver samples with liver cancer which were collected in US.

\section{Histology and immunohistochemistry}

Formalin-fixed, paraffin-embedded liver tissue samples were cut into $4 \mu \mathrm{m}$-thick sections and stained with hematoxylin eosin (H\&E), Sirius red, and immunohistochemistry (IHC) according to standard procedures. Fibrosis was scored according to the Ishak scoring system [29]. The amount of Sirius red 
staining was quantified with ImageJ. For IHC, liver sections were stained with the following antibodies: JAK1 (sc-1677; Santa Cruz Biotechnology), JAK2 (\#3230; Cell signaling Technology), Alpha-Smooth muscle actin (a-SMA,1A4, ab7817; Abcam), Both the intensity and extent of immunostaining were taken into consideration when analyzing the data. The intensity of staining was determined by the following rules: 0 for negative; 1 for weak staining; 2 for moderate staining; 3 for strong staining. The staining extent was determined by the following rules: 0 for no staining; 1 for less than $10 \% ; 2$ for $10 \%$ to $50 \%$; 3 for $51 \%$ to $75 \%$; 4 for more than $75 \%$. We randomly selected 5 areas from each area to count the intensity and extent of staining and to calculate the mean staining extent. The score was obtained by plus these two values (intensity score + extent score).

\section{Liver function assay}

Serum levels of several biochemical markers were measured to assess liver function and liver injury. The measured biochemical markers included alkaline phosphatase (ALP), alanine aminotransferase (ALT), aspartate aminotransferase (AST), total bilirubin (TBIL) and albumin (Alb) were measured using standard laboratory assays.

\section{Western blotting}

Western blotting was performed as previously described [30]. The primary antibodies were purchased from Santa Cruz Biotechnology [JAK1 (sc-1677)], Cell signaling Technology [JAK2 (\#3230), phosho-JAK1 (Tyr1022/1023, \#3331), 
phosho-JAK2 (Tyr1007/1008, \#3771), STAT3(\#9139), phosho-STAT3 (Tyr705,\#9145), phosho-STAT3 (Ser705, \#9134), STAT5 (\#94205), phoshoSTAT5 (Tyr694, \#9359)], Abcam [a-SMA (1A4, ab7817)], Bimake [PDGFR $\beta$ (A5541), PAI-1 (A5396)], Bioworld [ $\beta$-actin (\#64132)]. $\beta$-actin was used as a loading control for all blots.

\section{Quantitative Real-time RT-PCR}

Total RNA was extracted from cells and tissues using Trizol (Takara, Japan), and was reverse transcribed into cDNA according to the manufacturer's instructions, and the GAPDH gene was used as gene control. The relative gene expression ratio was calculated by the $\Delta \Delta \mathrm{Ct}$ method. Experiments were performed according to the manufacturer's instructions (Takara, Japan). Independent experiments were done in triplicate.

\section{Cell proliferation assay}

LX-2 cells were seeded at a density of 3000 cells/well in 96 -well microplates. The cells were treated with siRNA transfection or varying concentrations of Ruxolitinib as designed $(0.1-100 \mu \mathrm{M})$. Cell viability was measured using the Cell Counting Assay Kit-8 (CCK-8; Dojindo, Japan) according to the manufacturer's instructions. For each experimental condition, five parallel wells were assigned to each group. Experiments were performed in triplicate.

\section{Cell migration assay}

LX-2 cells were seeded in a 6-well plate treated with siRNA transfection or drug for 24h. Migration assays were conducted using 24-well Boyden chambers 
containing inserts ( $8 \mu \mathrm{m}$ pores; BD Biosciences,USA). The lower chamber was filled with medium containing $10 \%$ serum, whereas the top chamber contained $1 \times 10^{5}$ cells without serum. The plates were incubated at $37{ }^{\circ} \mathrm{C}$ in $5 \% \mathrm{CO}_{2}$ for 10h. After migration, the cells that had migrated to the underside of the membrane were fixed with paraformaldehyde and stained with $0.1 \%$ crystal violet. Migrated cells on each insert were counted in five randomly selected fields and quantified using the ImageJ software.

\section{Cell apoptosis assay}

LX-2 cells were seeded in 6-well plates and treated with different concentrations of Ruxolitinib for 24 hours. For cells were then harvested and stained with Annexin V-FITC Apoptosis Detection Kit (BD Pharmingen, CA) according to the manufacture's protocol. Experiments were performed in triplicate.

\section{Mouse models}

All animal experiments were conducted in accordance with the National Institutes of Health guide for the care and use of Laboratory animals and approved by the Animal Care and Use Committee of Southern Medical University.

\section{Liver fibrosis progression and reversal model induced by $\mathrm{CCl}_{4}$}

For the Liver fibrosis progression model, 4-6 weeks old male C57BL/6 mice (Vital River, Beijing, China) were treated three times a week with or without $0.1 \mathrm{ml}$ of a $40 \% \mathrm{CCl}_{4}$ in olive oil by oral gavage for 8 weeks and mice were treated with or without Ruxolitinib (30 mg/kg, oral gavage, each day) from 5 to 
8 weeks. For Liver fibrosis reversal model, 4-6 weeks old male C57BL/6 mice (Vital River, Beijing, China) were treated three times a week with or without $0.1 \mathrm{ml}$ of a $40 \% \mathrm{CCl}_{4}$ in olive oil by oral gavage for 6 weeks, and then mice allowed to recover form 6 to 8 weeks, with or without treatment with Ruxoltinib (30 mg/kg, oral gavage, each day).

\section{Panlobular liver fibrosis model induced by TAA}

6-8 weeks old male C57BL/6 mice were accepted an optimistic dose-escalating TAA as described [31]. And then mice were allowed to recover from 6 to 10 weeks, with or without treatment with Ruxolitinib $(30 \mathrm{mg} / \mathrm{kg}$, oral gavage, each day).

\section{Statistical analysis}

All statistical analyses were performed with GraphPad Prism V.5.00. Data are expressed as mean \pm standard deviation (SD). Differences between two groups were compared using a two-tailed unpaired Student's t-test. One-way ANOVA was used for sample comparison among multiple groups. Statistical significance was defined as $P<0.05$.

\section{Results}

Up-regulated JAK1 and JAK2 are associated with liver fibrosis and liver disease in human and mice

To evaluate whether JAK1 and JAK2 expression are associated with liver fibrosis/cirrhosis and liver cancer, IHC staining was used to examine JAK1 and 
JAK2 expression in human liver tissue array with confirmed cirrhosis and cancer. JAK1 and JAK2 expression were markedly higher in liver cirrhosis tissues $(n=22)$, while highest in liver cancer tissues $(n=53)$ compared to normal liver tissues ( $n=5)$ (Fig.1A and B). We further analyzed the relationship between the expression of JAK1 and JAK2 and the degree of liver fibrosis in mice. Sirius Red staining was used to confirm constructed liver fibrosis mouse model induced by $\mathrm{CCl}_{4}$ for 6 weeks or 8 weeks respectively, and IHC staining showed expression of JAK1 and JAK2 were a significant increase in fibrosis liver compared with controls (Fig.1C and D), which is constant to human liver disease. Our results suggest that the expression of JAK1 and JAK2 was associated with liver cancer progression and was also positively correlated with the severity of liver fibrosis.

Knockdown of JAK1 and JAK2 inhibits activation, proliferation and migration of HSCs

Since the activation and proliferation of HSCs in the main trigger for liver fibrosis and subsequent liver disease, to investigate the role of JAK1 and JAK2 on HSCs, LX-2 cells were transfected with siJAK1, siJAK2, or both of them. The transfected efficiency was confirmed by qRT-PCR and Western blotting (Fig.2A). As $\alpha$-SMA and platelet-derived growth factor receptor beta (PDGFR $\beta$ ) is the common marker for HSCs activation, the mRNA and protein level were detected to investigate the role of JAK1 and JAK2 on the activation of HSCs. As shown in Figure 2B, $\alpha$-SMA and PDGFR $\beta$ were significantly decreased in 
LX-2 cells transfected with siJAK1 or siJAK2. Furthermore, the lowest expression was found in cells co-transfected with siJAK1 and siJAK2.

Next, we analyzed the effect of JAK1 and JAK2 on the proliferation and migration of the HSCs. Knockdown of JAK1 or JAK2 had a similar impact on inhibiting proliferation, while the proliferation of HSCs was further decreased in the co-transfected group (Fig.2C). Likewise, the number of migrated HSCs was significantly reduced in cells with siJAK1 or siJAK2 and lowest in the cotransfected group compared to the control group (Fig.2D). Furthermore, we investigated the effect of JAK1 and JAK2 on the downstream signaling and revealed that expression of $\mathrm{p}$-JAK1, p-JAK2, STAT3, and p-STAT3 was significantly decreased (Fig.2E). These results indicate that knockdown JAK1 and JAK2 may inhibit the activation, proliferation and migration of HSCs.

\section{JAK1 and JAK2 antagonism has anti-fibrotic activity in vitro}

Ruxolitinib is a selective small-molecule oral inhibitor of JAK1/2, and it was verified to effectively inhibit JAK1 phosphorylation at Tyr1022/1023 and JAK2 phosphorylation at Tyr1007/1008, the critical activation site at the kinase domain of JAK1 and JAK2, respectively (Supporting Fig.S1). Moreover, we revealed that Ruxolitinib significantly decreased STAT3 phosphorylation at Tyr705 and Ser727, STAT5 phosphorylation at Tyr694, while no significant changes were observed in STAT3 and STAT5 (Fig.3A). Then we utilized Ruxolitinib to investigate whether JAK1 and JAK2 antagonism has anti-fibrotic activity. 
By using Ruxolitinib on LX-2 cells, we found Ruxolitinib inhibits cell proliferation in a dose and time-dependent manner in LX-2 cells (Fig.3B), and the halfmaximal inhibitory concentration (IC 50 ) of Ruxolitinib on LX-2 cell was $14.01 \mu \mathrm{M}$ (Fig.3C). Furthermore, Ruxolitinib significantly inhibited the expression of HSCs activation marker ( $\alpha-S M A$ and $P D G F R \beta)$ and collagen-associated marker (COL1A1 and PAI-1) (Fig.3D). Besides, Ruxolitinib also significantly inhibited the number of migrations (Fig.3E) and induced the level of apoptosis (Fig.3F) in LX-2 cells in a dose-dependent manner. These results demonstrate that Ruxolitinib, as the JAK1 and JAK2 antagonism, inhibits proliferation, activation, migration and promoted cell apoptosis of HSCs in vitro and may exert an antifibrotic activity by inhibiting phosphorylation site of JAK1/2 downstream signaling.

\section{Ruxolitinib attenuates the progression of liver pan-lobular fibrosis induced by $\mathrm{CCl}_{4}$}

To determine the appropriate dose of Ruxolitinib in vivo, we explored and conformed the appropriate drug concentration $(30 \mathrm{mg} / \mathrm{kg})$ in the preliminary experiment and verified it had no side effects on normal mice, which was used in the subsequent animal experiments (Supporting Fig.S2).

Progressive liver pan-lobular fibrosis was induced in C57BL/6 mice by repeated $\mathrm{CCl} 4$ oral gavage for up to 8 weeks, as described above. To evaluate the antifibrotic efficacy of Ruxolitinib on advanced liver fibrosis, we administered Ruxolitinib (30mg/kg, oral gavage, each day) concurrently with fibrosis 
induction, from 4 weeks to 8 weeks while vehicle control were administered in parallel (Fig.4A). Gross morphology and HE staining analysis revealed that Ruxolitinib significantly reduced liver fibrosis and further confirmed by Sirius red staining. Also, IHC staining showed expression of a-SMA was increased in mice with $\mathrm{CCl}_{4}$ while significantly decreased in mice treated with Ruxolitinib highlighting the inhibition of Ruxolitinib on the activation of HSCs (Fig.4B and C). Moreover, Western blotting showed that Ruxolitinib effectively inhibits the expression of p-JAK1, p-JAK2, and a-SMA (Fig.4D). To explore that the effect of Ruxolitinib on hepatic function, serum level of ALT, AST, TBIL, ALP, and Alb were examined. We found that Ruxolitinib treatment partially decreased the level of ALT, AST, and TBIL, which is increased by $\mathrm{CCl}_{4}$, indicating that it may partially protect the liver from damage (Fig.4E). These results suggest that Ruxolitinib attenuates $\mathrm{CCl}_{4}$-induced liver fibrosis by targeting $\mathrm{JAK} 1 / 2$ and activation of HSCs.

\section{Ruxolitinib accelerates the reversal of liver fibrosis in mice}

Since exploring effective intervention for reversing liver fibrosis in meaning for liver disease, we sought to test whether Ruxolitinib may promote liver fibrosis reversal in different mice models induced by $\mathrm{CCl}_{4}$ or TAA, which is widely used as a reversible model for study drug intervention [31]. As shown in Figure 5A, the liver fibrosis mice were induced by $\mathrm{CCl}_{4}$ for 6 weeks, and then mice were allowed to recover from 6 weeks to 8 weeks, with or without treatment with Ruxolitinib. Morphological analysis and tissue staining showed that the 
development of liver fibrosis and activation of HSCs in the mice treated with $\mathrm{CCl}_{4}$ for 6 weeks, while partially spontaneous reversion was found in 8-weekold mice without Ruxolitinib. Interestingly, the degree of liver fibrosis and the activation of HSCs were significantly reversed in 8-week-old mice treated with Ruxolitinib for 2 weeks which is confirmed by western blot (Fig.5B-D). Furthermore, the serum markers of hepatic function were examined, and as shown in Figure 5E, the levels of ALT, AST, and TBIL were significantly reduced in mice with Ruxolitinib compared that those without Ruxolitinib, indicating Ruxolitinib may promote the improvement of liver function in mice.

To confirm the reversal effect of Ruxolitinib, another liver fibrosis mice model induced by TAA was used and consistent results were found. As shown in Figure $6 \mathrm{~A}$, the mice were administered with TAA for 6 weeks, and then allowed to recover from 6 weeks to 10 weeks, with or without treatment with Ruxolitinib. Similarly, Ruxolitinib was found to significantly reversed the degree of liver fibrosis and the activation of HSCs (Fig.6B-D) and decreased the levels of ALT, AST and ALP in 10 weeks mice. In conclusion, Ruxolitinib accelerates reversal of liver fibrosis and improve the liver damage in different mice model induced by $\mathrm{CCl}_{4}$ or TAA.

\section{Discussion}

Liver fibrosis and cirrhosis are major health problems worldwide, causing more than 1 million deaths per year [32], for which there are currently no approved 
effective drugs [33]. Activated HSCs play a crucial role in liver fibrosis. However, the molecular mechanisms by which HSCs are activated and converted to a fibroblast phenotype are not fully understood. In this study, we found that enhanced JAK1 and JAK2 expression were associated with liver fibrosis/cirrhosis and liver cancer. IHC staining further demonstrated that the upregulation of JAK1 and JAK2 was mainly localized in hepatocytes and HSCs. More interestingly, we found that expression of JAK1 and JAK2 was positively correlated with the progression of liver cancer and the severity of liver fibrosis. Further, silencing of JAK1 and JAK2 down-regulated its downstream signaling and inhibited proliferation, activation, migration of HSCs. JAK1/2 inhibition had similar actions on HSCs in a concentration-dependent model in vitro and obviously attenuated the progress of liver fibrosis, promoted its reversal, and improved the liver damage in different liver fibrosis mice model induced by $\mathrm{CCl}_{4}$ or TAA. Therefore, JAK1/2 may be considered as a potential marker of activated HSCs and therapeutic targets in the treatment of liver fibrosis.

The JAK family is a class of non-receptor tyrosine kinases, play an important role in the development of many diseases, especially JAK2, which has been treated as a target of myeloproliferative diseases [34]. The existing reports suggests that STAT3 plays an important role in the development of liver fibrosis and demonstrates that STAT3 selective inhibitors can effectively attenuate the progression of liver fibrosis [21,35-37]. However, JAK1 and JAK2, which are upstream of STAT3, have been mainly demonstrated to play a role in blood 
system diseases such as myelofibrosis and lymphoma [22,23]. Interestingly, this study showed that JAK1/2 expression was up-regulated in liver fibrosis and $\mathrm{HCC}$, and further positively associated with liver cancer progression and the severity of liver fibrosis, indicating that JAK1/2 may take an action in liver fibrosis. Activated HSCs have been demonstrated to play a central role in liver fibrogenesis by producing most of the ECM. HSCs are quiescent, and the underlying mechanism of activation is still not clear. We found We found JAK1/2 promoted the proliferation and activation of HSCs in vitro. Take together, these results suggest that JAK $1 / 2$ promotes activation of HSCs and may be useful markers to monitor liver fibrosis and HCC development.

Subsequently evidence has demonstrated that Ruxolitinib had significant antifibrotic activity both in vitro and in vivo. Ruxolitinib is the most potent JAK1/2 inhibitor for blocking JAK1 phosphorylation at Tyr1022/1023 and JAK2 phosphorylation at Tyr1007/1008. A variety of chronic liver diseases lead to cirrhosis and HCC associated with high morbidity and mortality, while the treatments for advanced liver fibrosis and cirrhosis are still unsatisfactory. Therefore, understanding the mechanism and finding the effective drug to treat and reverse liver fibrosis are urgently needed [38]. Our date showed that Ruxolitinib significantly inhibited the activation of HSCs in vitro and suppressed liver fibrosis progression and accelerated reversal of liver fibrosis in independent murine models. We speculate that these phenomenon are mainly due to the anti-fibrotic and hepatoprotective effects of Ruxolitinib. However, the 
specific mechanism underlying these phenomenon are not fully elucidated. Further studies to explore the mechanism by primary HSCs and to investigate whether JAK1/2 inhibition prevents recurrence of $\mathrm{HCC}$ with the background of liver fibrosis in needed.

More interestingly, we observed that Ruxolitinib has a good effect on improving liver function in different liver fibrosis models, especially improving acute liver injury such as transaminase and bilirubin, which caused our concern. Patients with advanced cirrhosis and liver cancer miss effective treatment opportunities due to the accompanying liver function damage. Ruxolitinib has been shown to significantly improve acute liver injury, reduce transaminase and bilirubin, and achieve the purpose of improving liver function, which may win the opportunity for HCC patients to get anti-tumor treatments.

To date, there are no efficient anti-fibrotic therapies available for chronic liver disease and HCC. Innovative medical treatments to stop or even reverse fibrosis are urgently needed. Therefore, JAK1 and JAK2 might play a key role in the process of liver fibrogenesis, and its potential inhibitor, Ruxolitinib, may be clinically useful in preventing or treating liver fibrosis (Fig.7).

\section{Conclusions}

In conclusion, JAK1/2 regulate the biological function of HSCs, highlighting its role in liver fibrosis and early prevention of HCC development and its inhibitor, Ruxolitinib, may be an effective drug for preventing and reversing liver fibrosis. 
Abbreviations: Alb, albumin; ALP, Alkaline phosphatase; ALT, Alanine aminotransferase; a-SMA, Alpha-Smooth muscle actin; AST, Aspartate aminotransferase; $\mathrm{CCl} 4$, Carbon tetrachloride; COL1A1, Collagen type I alpha 1; ECM, Extracellular matrix; FDA, Food and Drug Administration; HCC, Hepatocellular carcinoma; H\&E, Hematoxylin-eosin; HSCs, Hepatic stellate cells; IC50, half maximal inhibitory concentration; IHC, immunohistochemistry; JAK, Janus kinase; JAK/STAT, Janus kinase/Signal Transducer and Activator of Transcription; NASH, nonalcoholic steatohepatitis; PAI-1, Plasminogen activator Inhibitor-1; PDGFRß, Platelet-derived growth factor receptor beta; siRNA, small interfering RNA; SSc, Systemic sclerosis; TAA, Thioacetamide; TBIL, total bilirubin

\section{Declarations}

\section{Ethics approval and consent to participate}

All animal experiments were conducted in accordance with the National Institutes of Health guide for the care and use of Laboratory animals and approved by the Animal Care and Use Committee of Southern Medical University.

\section{Availability of data and materials}

The datasets generated and/or analyzed during the current study are not publicly available but are available from the corresponding author upon 
reasonable request.

\section{Competing interests}

All authors declare no conflicts of interest.

\section{Funding}

This work was supported by the Project of Administration of Traditional Chinese Medicine of Guangdong Province of China (NO. 20203006); Science and Technology Program of Guangzhou, China (NO. 202002030075); President's fund of Integrated hospital of traditional Chinese Medicine (NO. 1201902004).

\section{Authors' contributions}

Zhenghui Song and Xinhui Liu designed the experiments, wrote the manuscript, and participated in most of the experiments. Yue Luo, Yun Liu assisted in cell biological experiments. Wan Zhang and Guanqi Dai assisted in animal studies. Hua Xiao revised the manuscript. Aimin $\mathrm{Li}$ and Jian Hong conceived and supervised the project.

\section{References}

1. Higashi T, Friedman SL, Hoshida Y. Hepatic stellate cells as key target in liver fibrosis. Adv Drug Deliv Rev 2017; 121: 27-42.

2. Qu C, Zheng D, Li S, Liu Y, Lidofsky A, Holmes JA, et al. Tyrosine kinase SYK is a potential therapeutic target for liver fibrosis. Hepatology 2018; 68(3): 1125-39.

3. Tsochatzis EA, Bosch J, Burroughs AK. Liver cirrhosis. Lancet 2014; 383(9930): 1749-61.

4. Wynn TA, Ramalingam TR. Mechanisms of fibrosis: therapeutic translation 
for fibrotic disease. Nature medicine 2012; 18(7): 1028-40.

5. Cohen-Naftaly M, Friedman SL. Current status of novel antifibrotic therapies in patients with chronic liver disease. Therap Adv Gastroenterol 2011; 4(6): 391-417.

6. Bataller R, Brenner DA. Liver fibrosis. J Clin Invest 2005; 115(2): 209-18.

7. Fuchs BC, Hoshida Y, Fujii T, Wei L, Yamada S, Lauwers GY, et al. Epidermal growth factor receptor inhibition attenuates liver fibrosis and development of hepatocellular carcinoma. Hepatology (Baltimore, Md) 2014; 59(4): 1577-90.

8. Chen L, Li J, Zhang J, Dai C, Liu X, Wang J, et al. S100A4 promotes liver fibrosis via activation of hepatic stellate cells. $J$ Hepatol 2015; 62(1): 156-64.

9. Friedman SL. Hepatic stellate cells: protean, multifunctional, and enigmatic cells of the liver. Physiological reviews 2008; 88(1): 125-72.

10. Krizhanovsky V, Yon M, Dickins RA, Hearn S, Simon J, Miething C, et al. Senescence of activated stellate cells limits liver fibrosis. Cell 2008; 134(4): 657-67.

11. Drew L. Liver cirrhosis: scar wars. Nature 2018; 564(7736): S73.

12. Ikenaga N, Peng ZW, Vaid KA, Liu SB, Yoshida S, Sverdlov DY, et al. Selective targeting of lysyl oxidase-like 2 (LOXL2) suppresses hepatic fibrosis progression and accelerates its reversal. 2017; 66(9): 1697-708.

13. Priceman SJ, Kujawski M, Shen S, Cherryholmes GA, Lee H, Zhang C, et al. Regulation of adipose tissue $T$ cell subsets by Stat3 is crucial for dietinduced obesity and insulin resistance. Proceedings of the National Academy of Sciences of the United States of America 2013; 110(32): 13079-84.

14. Deng J, Liu Y, Lee H, Herrmann A, Zhang W, Zhang C, et al. S1PR1-STAT3 signaling is crucial for myeloid cell colonization at future metastatic sites. Cancer cell 2012; 21(5): 642-54.

15. Park EJ, Lee JH, Yu GY, He G, Ali SR, Holzer RG, et al. Dietary and genetic obesity promote liver inflammation and tumorigenesis by enhancing IL-6 and 
TNF expression. Cell 2010; 140(2): 197-208.

16. Carro MS, Lim WK, Alvarez MJ, Bollo RJ, Zhao X, Snyder EY, et al. The transcriptional network for mesenchymal transformation of brain tumours. Nature 2010; 463(7279): 318-25.

17. Marotta LL, Almendro V, Marusyk A, Shipitsin M, Schemme J, Walker SR, et al. The JAK2/STAT3 signaling pathway is required for growth of CD44(+)CD24(-) stem cell-like breast cancer cells in human tumors. The Journal of clinical investigation 2011; 121(7): 2723-35.

18. Schroeder A, Herrmann A, Cherryholmes G, Kowolik C, Buettner R, Pal S, et al. Loss of androgen receptor expression promotes a stem-like cell phenotype in prostate cancer through STAT3 signaling. Cancer Res 2014; 74(4): 1227-37.

19. Yu H, Lee H, Herrmann A, Buettner R, Jove R. Revisiting STAT3 signalling in cancer: new and unexpected biological functions. Nat Rev Cancer 2014; 14(11): 736-46.

20. Kong X, Horiguchi N, Mori M, Gao B. Cytokines and STATs in Liver Fibrosis. Frontiers in physiology 2012; 3: 69.

21. Deng YR, Ma HD, Tsuneyama K, Yang W, Wang YH, Lu FT, et al. STAT3mediated attenuation of $\mathrm{CCl} 4$-induced mouse liver fibrosis by the protein kinase inhibitor sorafenib. Journal of autoimmunity 2013; 46: 25-34.

22. Zhang Y, Liang R, Chen CW, Mallano T, Dees C, Distler A, et al. JAK1dependent transphosphorylation of JAK2 limits the antifibrotic effects of selective JAK2 inhibitors on long-term treatment. Annals of the rheumatic diseases 2017; 76(8): 1467-75.

23. Chakraborty D, Šumová B, Mallano T, Chen CW, Distler A, Bergmann C, et al. Activation of STAT3 integrates common profibrotic pathways to promote fibroblast activation and tissue fibrosis. Nat Commun 2017; 8(1): 1130.

24. Verstovsek S, Mesa RA, Gotlib J, Levy RS, Gupta V, DiPersio JF, et al. A double-blind, placebo-controlled trial of ruxolitinib for myelofibrosis. $N$ Engl $J$ 
Med 2012; 366(9): 799-807.

25. Harrison C, Kiladjian JJ, Al-Ali HK, Gisslinger H, Waltzman R, Stalbovskaya $\mathrm{V}$, et al. JAK inhibition with ruxolitinib versus best available therapy for myelofibrosis. N Engl J Med 2012; 366(9): 787-98.

26. Quintás-Cardama A, Vaddi K, Liu P, Manshouri T, Li J, Scherle PA, et al. Preclinical characterization of the selective JAK1/2 inhibitor INCB018424: therapeutic implications for the treatment of myeloproliferative neoplasms. Blood 2010; 115(15): 3109-17.

27. Vannucchi AM, Kiladjian JJ, Griesshammer M, Masszi T, Durrant S, Passamonti $F$, et al. Ruxolitinib versus standard therapy for the treatment of polycythemia vera. The New England journal of medicine 2015; 372(5): 426-35. 28. Scott LM, Gandhi MK. Deregulated JAK/STAT signalling in lymphomagenesis, and its implications for the development of new targeted therapies. Blood Rev 2015; 29(6): 405-15.

29. Ding N, Yu RT, Subramaniam N, Sherman MH, Wilson C, Rao R, et al. A vitamin $\mathrm{D}$ receptor/SMAD genomic circuit gates hepatic fibrotic response. Cell 2013; 153(3): 601-13.

30. Hong J, Hu K, Yuan Y, Sang Y, Bu Q, Chen G, et al. CHK1 targets spleen tyrosine kinase $(\mathrm{L})$ for proteolysis in hepatocellular carcinoma. The Journal of clinical investigation 2012; 122(6): 2165-75.

31. Popov Y, Sverdlov DY, Sharma AK, Bhaskar KR, Li S, Freitag TL, et al. Tissue transglutaminase does not affect fibrotic matrix stability or regression of liver fibrosis in mice. Gastroenterology 2011; 140(5): 1642-52.

32. Mokdad AA, Lopez AD, Shahraz S, Lozano R, Mokdad AH, Stanaway J, et al. Liver cirrhosis mortality in 187 countries between 1980 and 2010: a systematic analysis. BMC Med 2014; 12: 145.

33. Troeger JS, Mederacke I, Gwak GY, Dapito DH, Mu X, Hsu CC, et al. Deactivation of hepatic stellate cells during liver fibrosis resolution in mice. Gastroenterology 2012; 143(4): 1073-83.e22. 
34. Rumi E, Cazzola M. Diagnosis, risk stratification, and response evaluation in classical myeloproliferative neoplasms. 2017; 129(6): 680-92.

35. Choi S, Jung HJ, Kim MW, Kang JH, Shin D, Jang YS, et al. A novel STAT3 inhibitor, STX-0119, attenuates liver fibrosis by inactivating hepatic stellate cells in mice. Biochemical and biophysical research communications 2019.

36. Nunez Lopez O, Bohanon FJ, Wang X, Ye N, Corsello T, Rojas-Khalil Y, et al. STAT3 Inhibition Suppresses Hepatic Stellate Cell Fibrogenesis: HJC0123, a Potential Therapeutic Agent for Liver Fibrosis. Laboratory investigation; a journal of technical methods and pathology 2016; 6(102): 100652-63.

37. Wang Z, Li J, Xiao W, Long J. The STAT3 inhibitor S3I-201 suppresses fibrogenesis and angiogenesis in liver fibrosis. 2018; 98(12): 1600-13.

38. Lee UE, Friedman SL. Mechanisms of hepatic fibrogenesis. Best practice \& research Clinical gastroenterology 2011; 25(2): 195-206.

\section{Figure legends}

Fig.1. Up-regulated JAK1 and JAK2 are associated with liver fibrosis and liver disease in human and mice. (A) JAK1 and JAK2 immunohistochemistry (IHC) was performed in human liver tissues microarray of normal, cirrhosis, and cancer (magnification 100X). (B) JAK1 and JAK2 scores quantified by IHC in normal liver, liver cirrhosis, and liver cancer. (C) The IHC and Sirius red staining of JAK1 and JAK2 were performed in mouse normal and fibrosis for 6 weeks $\mathrm{CCl}_{4}$-induced or 8 weeks $\mathrm{CCl}_{4}$-induced liver tissues (magnification $100 \mathrm{X}$ and 200X). (D) JAK1 and JAK2 scores quantified by IHC in normal liver, 6 weeks $\mathrm{CCl}_{4}$-induced liver fibrosis and 8 weeks $\mathrm{CCl}_{4}$-induced liver fibrosis. Data presented are means $\pm \mathrm{SD} .{ }^{* *}, P<0.01 ;{ }^{* * *}, P<0.001$.

Fig.2. Knockdown of JAK1 and JAK2 inhibits activation, proliferation and 
migration of HSCs. (A) The left panel shows LX-2 cells co-interfered with siJAK1 and siJAK2 reduced JAK1 and JAK2 mRNA expression. The right panel shows that siJAK2 reduced JAK1 and JAK2 protein expression in LX-2 cells cointerfered with siJAK1. (B) The left panel shows that knockdown JAK1 and JAK2 reduced mRNA expression of ACTA2, PDGFR $\beta$ in LX-2 cells. The right panel shows knockdown JAK1 and JAK2 reduced protein expression of $\alpha$-SMA, PDGFRß. (C) Knockdown expression of JAK1 and JAK2 inhibited LX-2 cell proliferation. (D) The left panel shows knockdown JAK1 and JAK2 inhibited migration (magnification 100X). The right shows the number of migrations counted after interfered with JAK1 and JAK2 in LX-2 cells. (E) LX-2 cells knockdown JAK1 and JAK2 reduced protein expression of p-JAK1 (Tyr1022/1023), p-JAK2 (Tyr1007/1008), STAT3, p-STAT3 (Tyr705), p-STAT3 (Ser727). Date presented are means $\pm \mathrm{SD} .{ }^{*}, P<0.05 ;{ }^{* *}, P<0.01 ;{ }^{* * *}, P<$ 0.001 .

Fig.3. JAK1 and JAK2 antagonism had anti-fibrotic activity in vitro. (A) The protein levels of JAK downstream in LX-2 cells treated with different concentrations of Ruxolitinib. (B) Ruxolitinib inhibited cell proliferative effect in LX-2 cells in a dose and time-dependent manner. (C) The half-maximal inhibitory concentration ( $\left.\mathrm{IC}_{50}\right)$ value of Ruxolitinib on LX-2 cells. (D) The upper panel shows relative mRNA expression of ACTA2, PDGFR $\beta$, COL1A1, and PAI1 in LX-2 cells treated with different concentrations of Ruxolitinib. The below shows protein expression of $\alpha-S M A$, PDGFR $\beta$, and PAI-1. (E) The left panel 
shows that treatment with Ruxolitinib inhibited LX-2 cell proliferation (magnification 100X). The right panel shows the number of migration counted in LX-2 cells treated with different concentrations of Ruxolitinib. (F) The left panel shows that treatment with Ruxolitinib promoted LX-2 cells apoptosis. The right panel shows the apoptosis rate of LX-2 cells treated with different concentrations of Ruxolitinib. Data presented are means \pm SD. NS $=$ not significant; ${ }^{*}, P<0.05 ;{ }^{* *}, P<0.01 ;{ }^{* * *}, P<0.001$.

Fig.4. Ruxolitinib attenuates the progression of liver pan-lobular fibrosis induced by $\mathrm{CCl}_{4}$. (A) Schematic of the experimental design of Ruxolitinib treatment in a $\mathrm{CCl}_{4}$-induced fibrosis progression model in mice. (B) Representative images of mouse livers stained with $H \& E$, Sirius red, and $\alpha-$ SMA antibodies. (C) Ruxolitinib reduced Ishak fibrosis score, and Sirius red, aSMA IHC staining (magnification 100X). (D) Ruxolitinib reduced the protein expression of p-JAK1, p-JAK2, and $\alpha$-SMA. (E) Serum levels of ALP, ALT, TBIL, ALP and Alb. Data presented are means \pm SD. NS = not significant; ${ }^{* *}, P<0.01$; ${ }^{* * *}, P<0.001$.

Fig.5. Ruxolitinib accelerates the reversal of liver fibrosis in $\mathrm{CCl}_{4}$ mouse model. (A) Schematic of the experimental design of Ruxolitinib treatment in a $\mathrm{CCl}_{4}$-induced fibrosis reversal model in mice. (B) Representative images of mouse livers stained with H\&E, Sirius red and a-SMA antibodies. (C) Ruxolitinib reduced Ishak fibrosis score, and Sirius red, a-SMA IHC staining (magnification 100X). (D) Ruxolitinib reduced the protein expression of p-JAK1, p-JAK2, and 
a-SMA. (E) Serum levels of ALP, ALT, TBIL, ALP, and Alb. Date presented are means \pm SD. NS $=$ not significant; ${ }^{*}, P<0.05 ;{ }^{* *}, P<0.01 ;{ }^{* * *}, P<0.001$.

Fig.6. Ruxolitinib accelerates the reversal of liver fibrosis in the TAA mouse model. (A) Schematic of the experimental design of Ruxolitinib treatment in a TAA-induced fibrosis reversal model in mice. (B) Representative images of mouse livers stained with H\&E, Sirius red and a-SMA antibodies. (C) Ruxolitinib reduced Ishak fibrosis score, and Sirius red, a-SMA IHC staining (magnification 100X). (D) Ruxolitinib reduced the protein expression of $p$-JAK1, p-JAK2, and a-SMA. (E) Serum levels of ALP, ALT, TBIL, ALP and Alb. Data presented are means $\pm \mathrm{SD}$. NS $=$ not significant; ${ }^{*}, P<0.05 ;{ }^{* *}, P<0.01 ;{ }^{* * *}, P$ $<0.001$

Fig.7. Proposed model of Ruxolitinib suppresses liver fibrosis progression and accelerates fibrosis reversal via selectively targeting JAK $1 / 2$. 
A
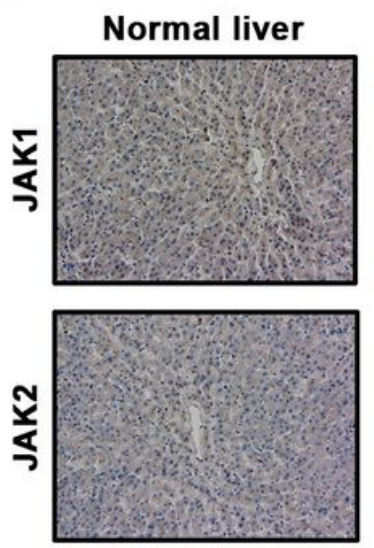

C
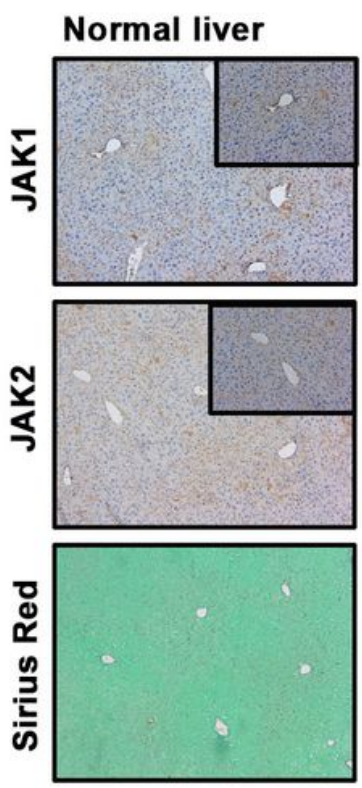

Liver cirrhosis
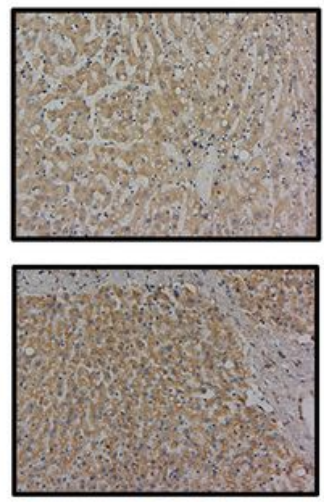

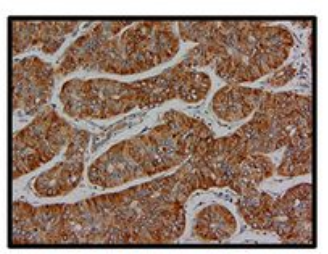

\section{Liver cancer}

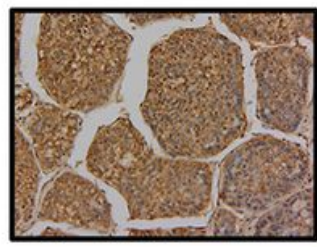

B
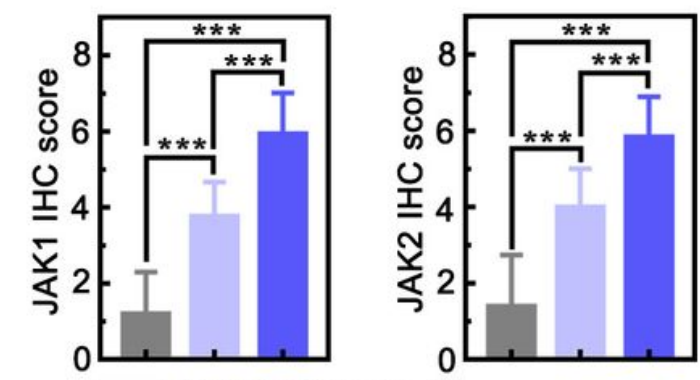

Normal liver $(n=5)$

Liver cirrhosis $(n=22)$

Liver cancer $(n=48)$

D

Liver fibrosis, 6 w
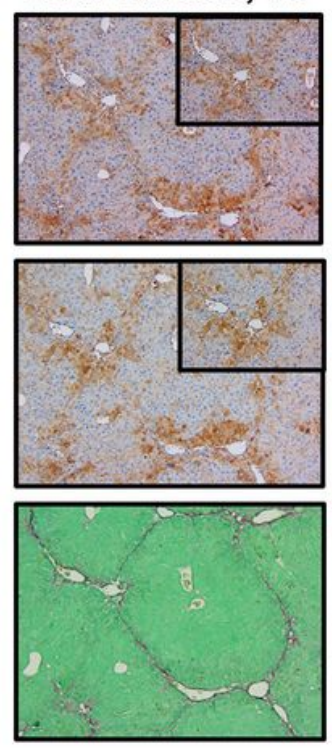

Liver fibrosis, 8 w
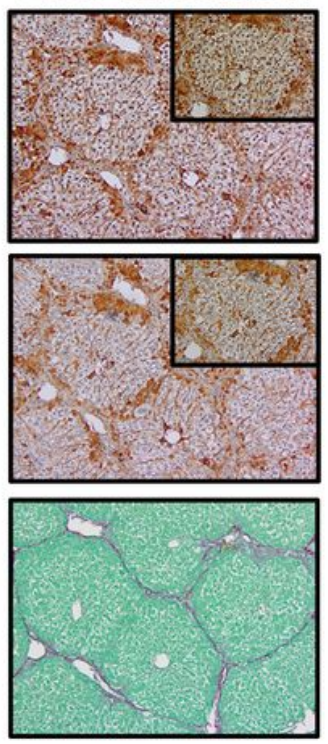
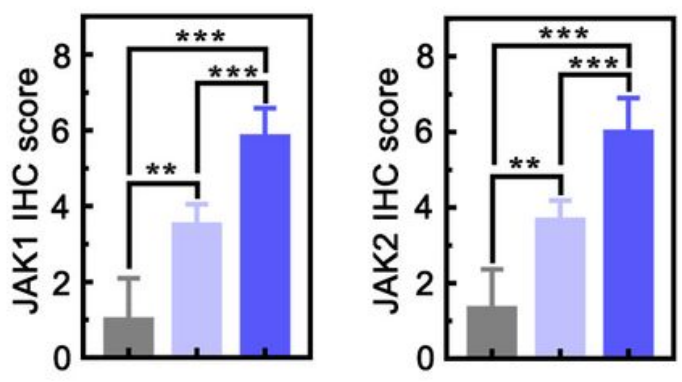

Normal liver $(n=6)$

Liver fibrosis, $6 w(n=8)$

Liver fibrosis, $8 w(n=8)$

Figure 1

Up-regulated JAK1 and JAK2 are associated with liver fibrosis and liver disease in human and mice. (A) JAK1 and JAK2 immunohistochemistry (IHC) was performed in human liver tissues microarray of normal, cirrhosis, and cancer (magnification 100X). (B) JAK1 and JAK2 scores quantified by IHC in normal liver, liver cirrhosis, and liver cancer. (C) The IHC and Sirius red staining of JAK1 and JAK2 were performed in mouse normal and fibrosis for 6 weeks $\mathrm{CCl} 4$-induced or 8 weeks $\mathrm{CCl} 4$-induced liver tissues (magnification 100X and 200X). (D) JAK1 and JAK2 scores quantified by IHC in normal liver, 6 weeks CCI4-induced liver fibrosis and 8 weeks CCl4-induced liver fibrosis. Data presented are means $\pm S D$. **, $\mathrm{P}<0.01$; ***, $\mathrm{P}<$ 0.001 . 


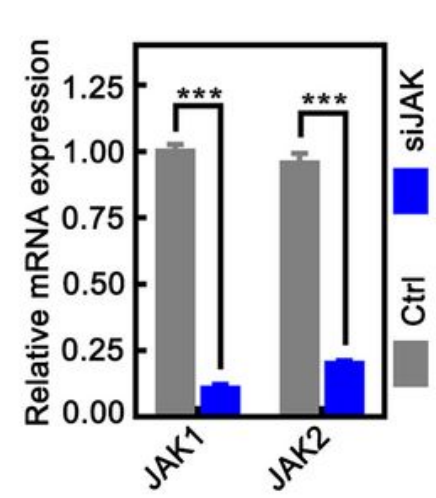

B

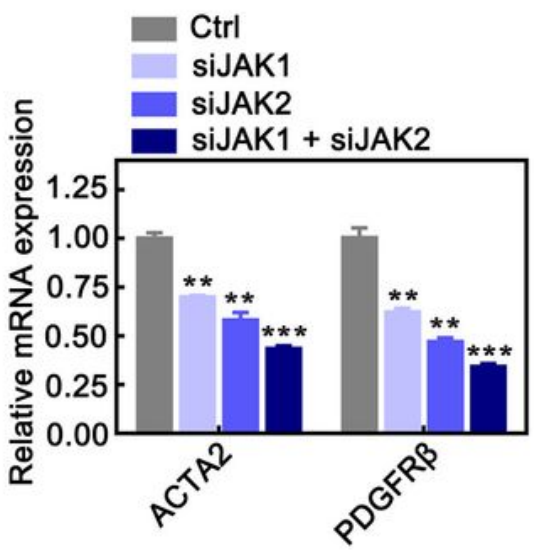

D
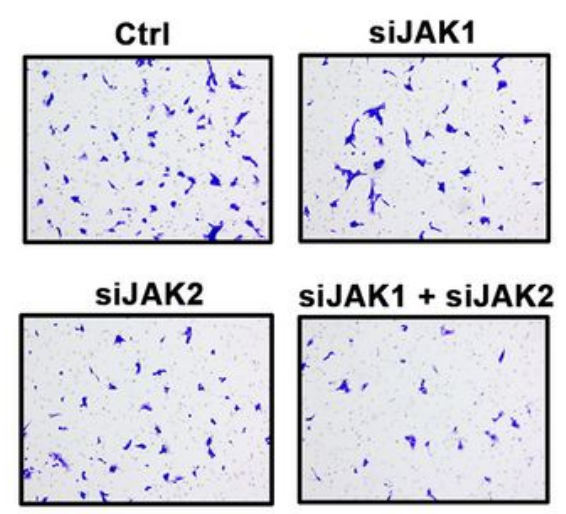
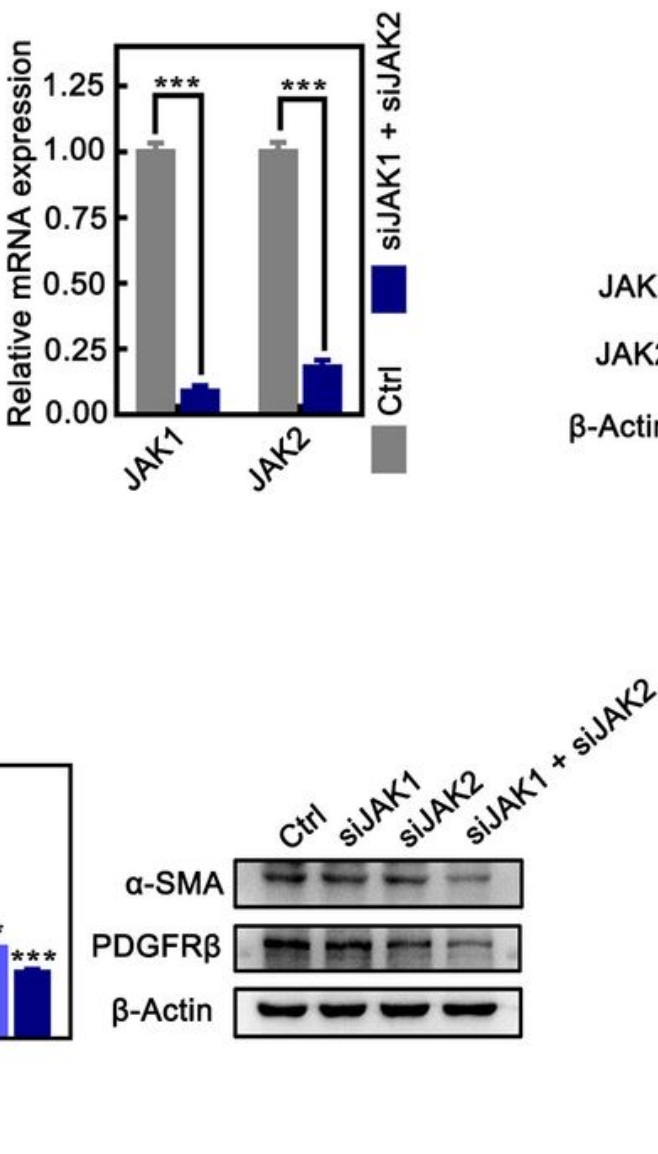

$\mathbf{E}$

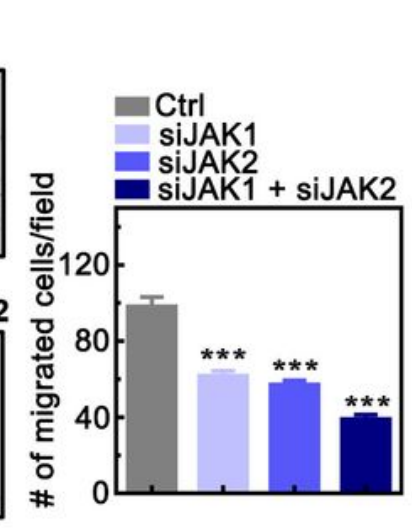

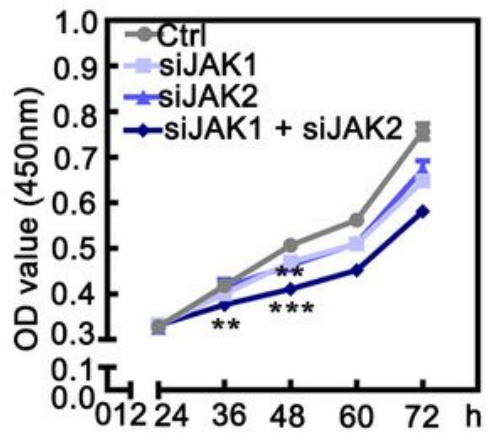

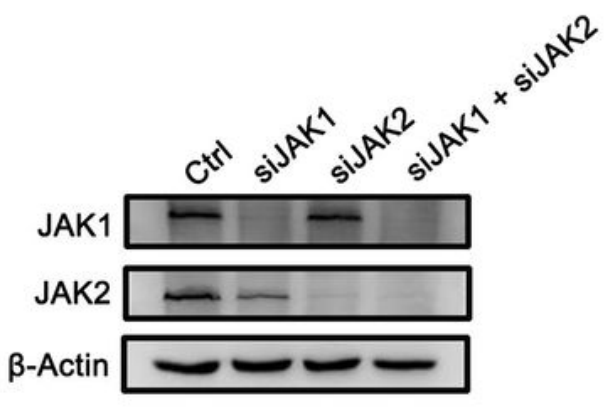

C

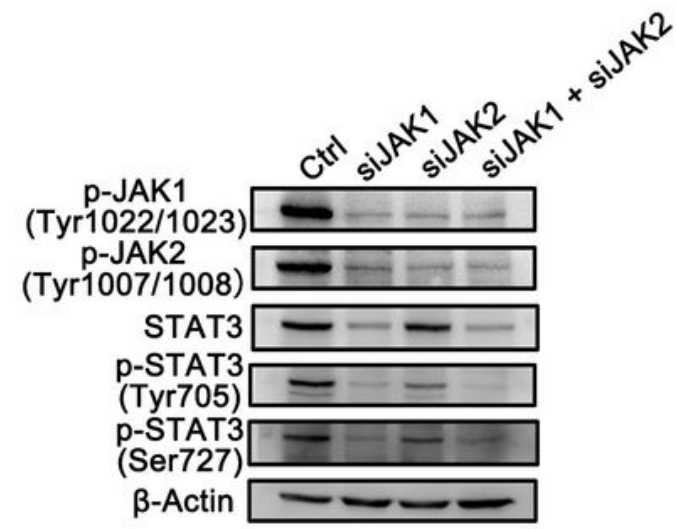

Figure 2

Knockdown of JAK1 and JAK2 inhibits activation, proliferation and migration of HSCs. (A) The left panel shows LX-2 cells co-interfered with siJAK1 and siJAK2 reduced JAK1 and JAK2 mRNA expression. The right panel shows that siJAK2 reduced JAK1 and JAK2 protein expression in LX-2 cells co-interfered with siJAK1. (B) The left panel shows that knockdown JAK1 and JAK2 reduced mRNA expression of ACTA2, PDGFR $\beta$ in LX-2 cells. The right panel shows knockdown JAK1 and JAK2 reduced protein expression of a- 
SMA, PDGFRß. (C) Knockdown expression of JAK1 and JAK2 inhibited LX-2 cell proliferation. (D) The left panel shows knockdown JAK1 and JAK2 inhibited migration (magnification 100X). The right shows the number of migrations counted after interfered with JAK1 and JAK2 in LX-2 cells. (E) LX-2 cells knockdown JAK1 and JAK2 reduced protein expression of p-JAK1 (Tyr1022/1023), p-JAK2

(Tyr1007/1008), STAT3, p-STAT3 (Tyr705), p-STAT3 (Ser727). Date presented are means \pm SD. *, $P<0.05$; $* \star, P<0.01 ; * \star *, P<0.001$.

A

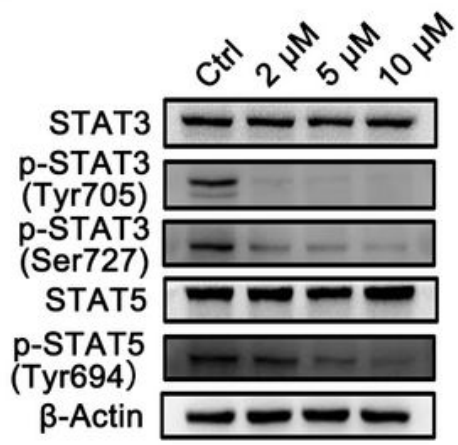

D

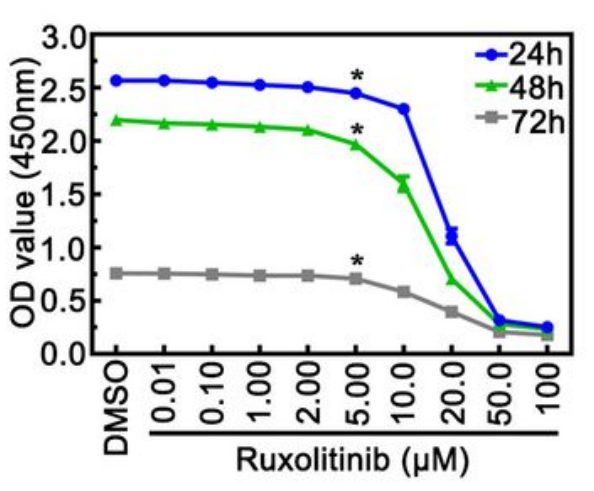

C

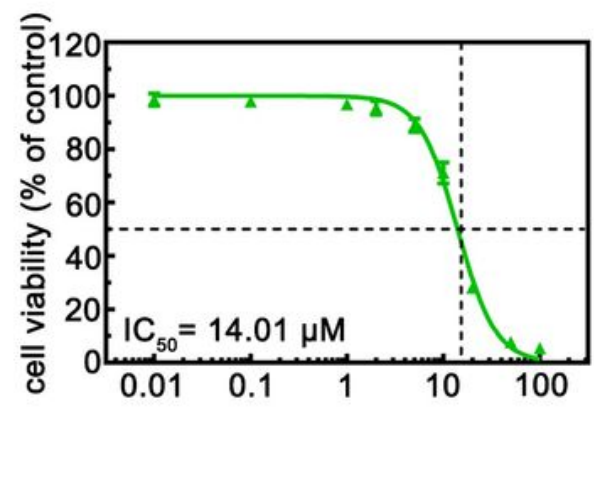

E
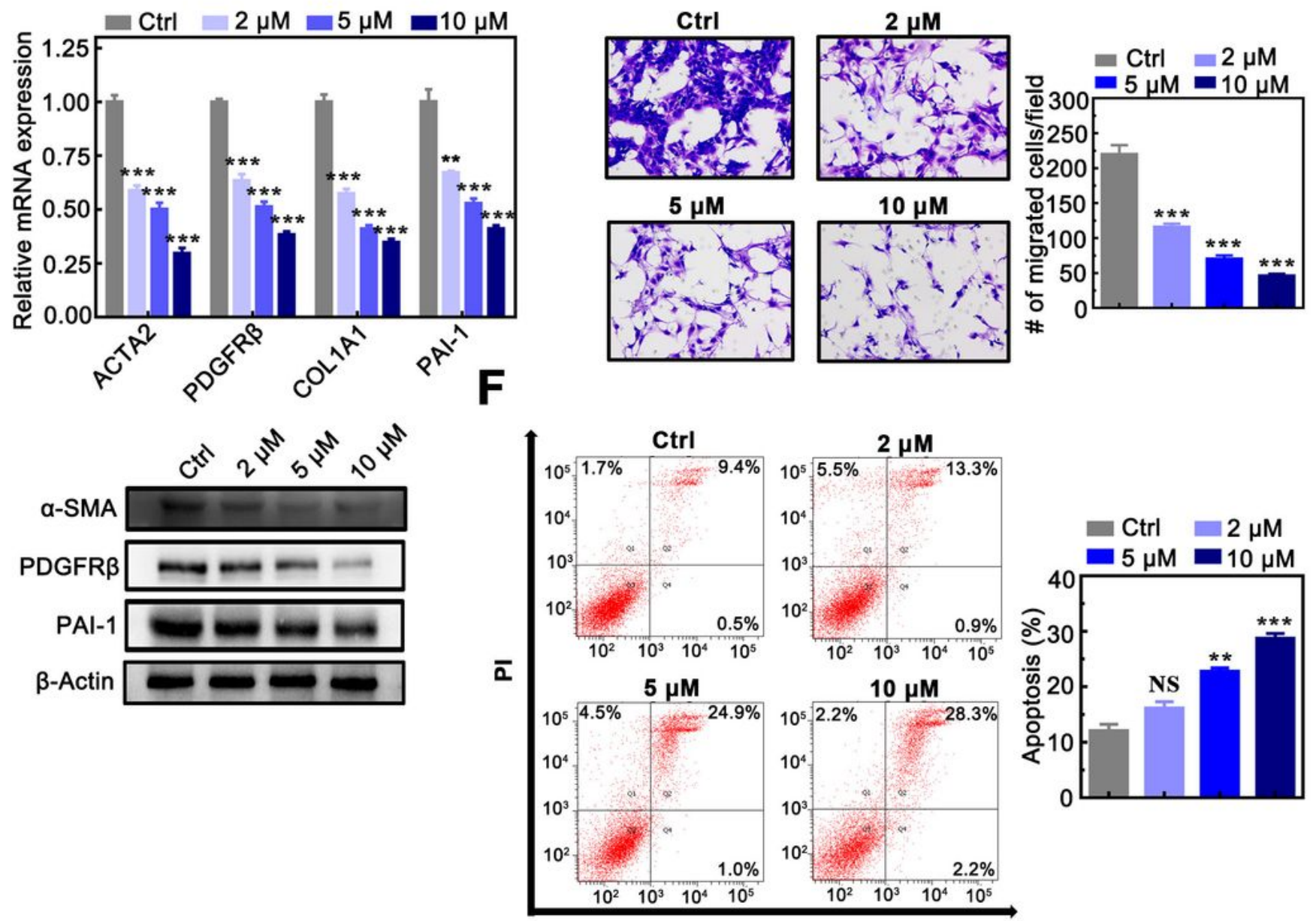

FITC-Annexin V

Figure 3 
JAK1 and JAK2 antagonism had anti-fibrotic activity in vitro. (A) The protein levels of JAK downstream in LX-2 cells treated with different concentrations of Ruxolitinib. (B) Ruxolitinib inhibited cell proliferative effect in LX-2 cells in a dose and time-dependent manner. (C) The half-maximal inhibitory concentration (IC50) value of Ruxolitinib on LX-2 cells. (D) The upper panel shows relative mRNA expression of ACTA2, PDGFRß, COL1A1, and PAI-1 in LX-2 cells treated with different concentrations of Ruxolitinib. The below shows protein expression of a-SMA, PDGFR $\beta$, and PAI-1. (E) The left panel shows that treatment with Ruxolitinib inhibited LX-2 cell proliferation (magnification 100X). The right panel shows the number of migration counted in LX-2 cells treated with different concentrations of Ruxolitinib. (F) The left panel shows that treatment with Ruxolitinib promoted LX-2 cells apoptosis. The right panel shows the apoptosis rate of LX-2 cells treated with different concentrations of Ruxolitinib. Data presented are means \pm SD. NS = not significant; ${ }^{*}, P<0.05 ; * \star, P<0.01 ; \star \star \star, ~ P<0.001$. 
A

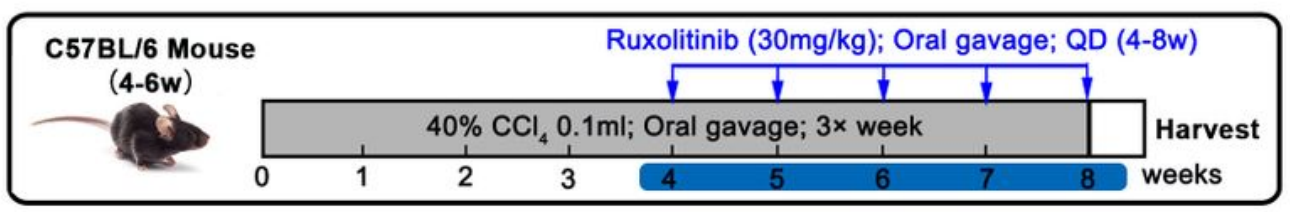

B
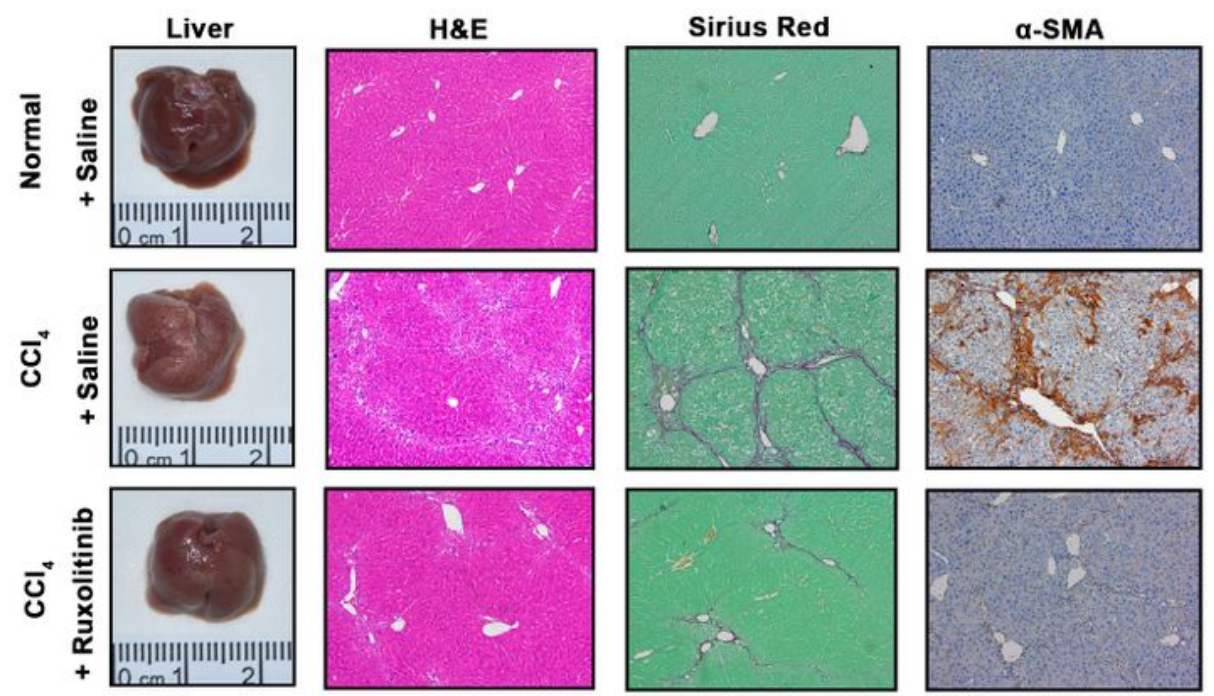

C

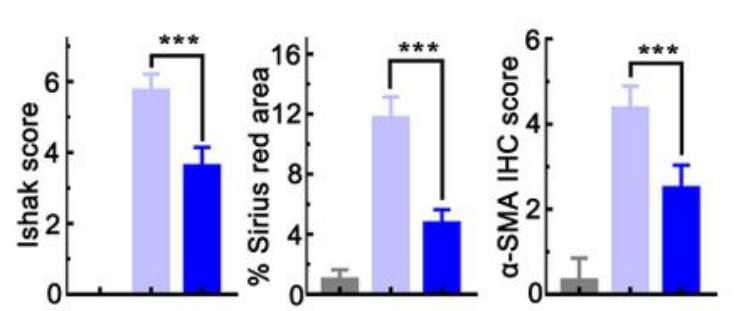

D

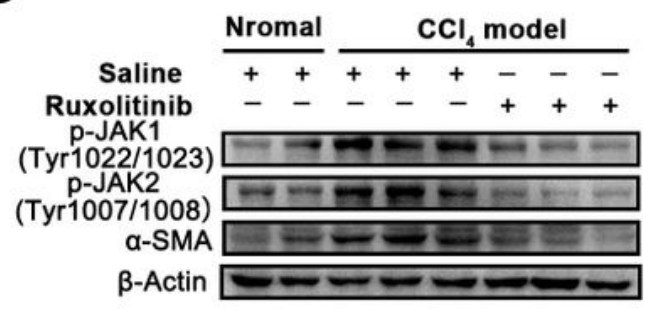

E

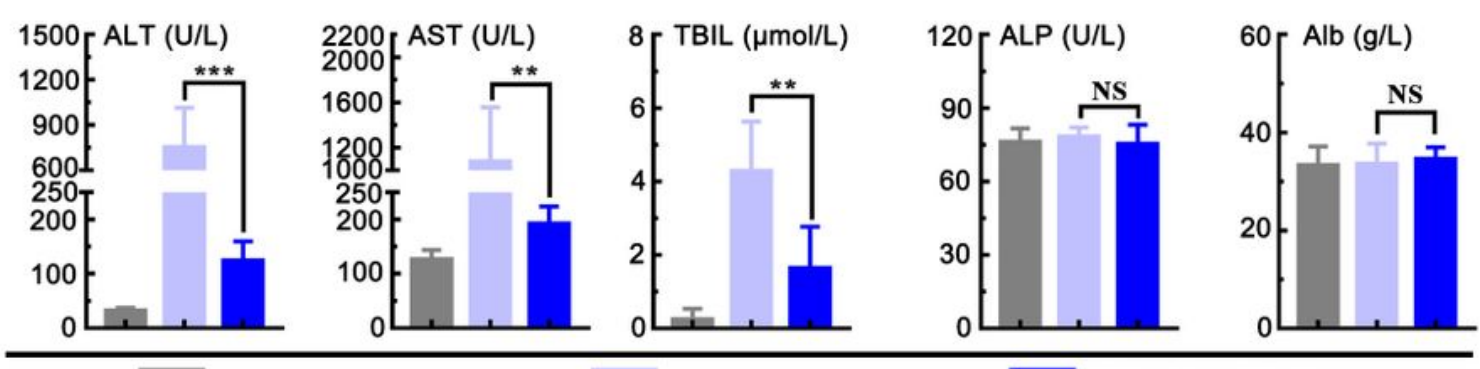

Normal + Saline

$\mathrm{CCl}_{4}+$ Saline

$\mathrm{CCl}_{4}+$ Ruxolitinib

\section{Figure 4}

Ruxolitinib attenuates the progression of liver pan-lobular fibrosis induced by $\mathrm{CCl} 4$. (A) Schematic of the experimental design of Ruxolitinib treatment in a $\mathrm{CCl} 4$-induced fibrosis progression model in mice. (B) Representative images of mouse livers stained with H\&E, Sirius red, and a-SMA antibodies. (C) Ruxolitinib reduced Ishak fibrosis score, and Sirius red, a-SMA IHC staining (magnification 100X). (D) Ruxolitinib 
reduced the protein expression of p-JAK1, p-JAK2, and a-SMA. (E) Serum levels of ALP, ALT, TBIL, ALP and Alb. Data presented are means \pm SD. NS = not significant; **, $P<0.01 ; * \star *, P<0.001$.

A

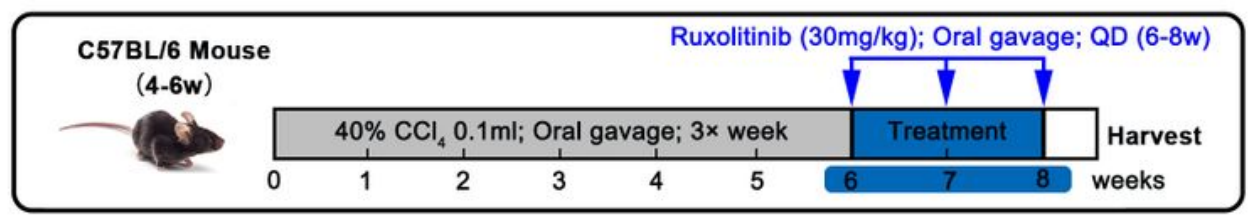

B
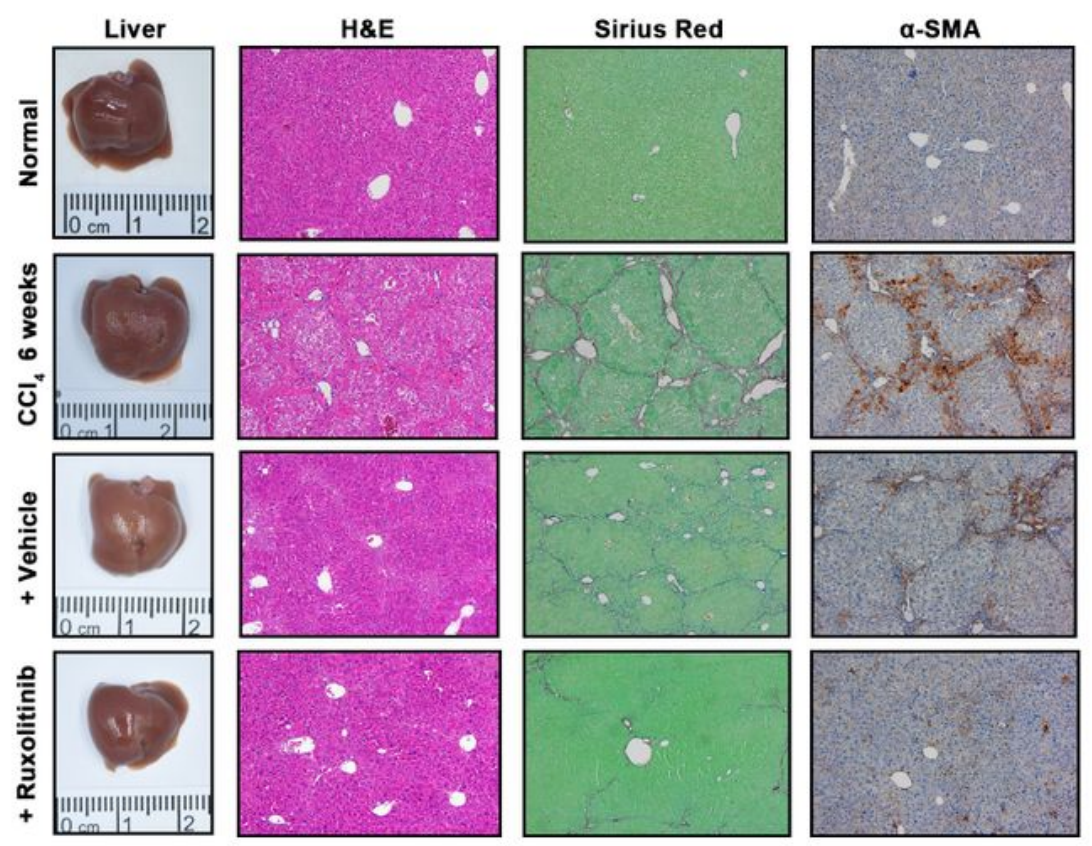

C

D

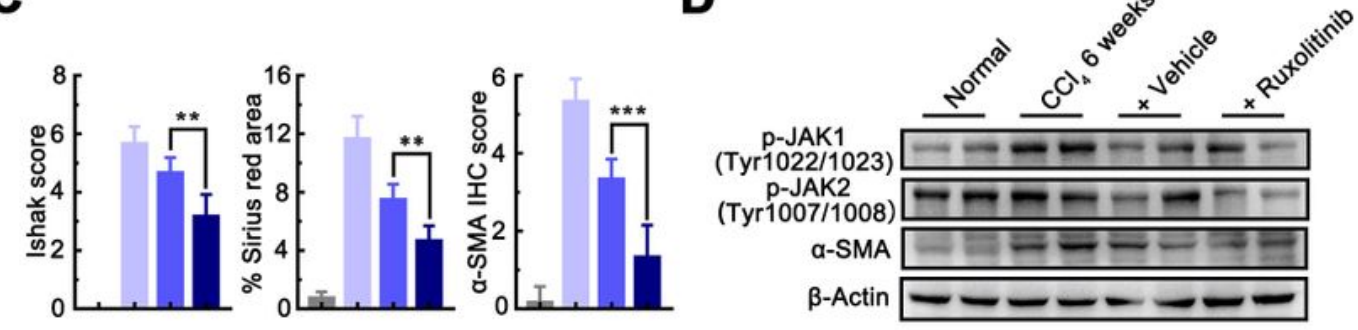

E

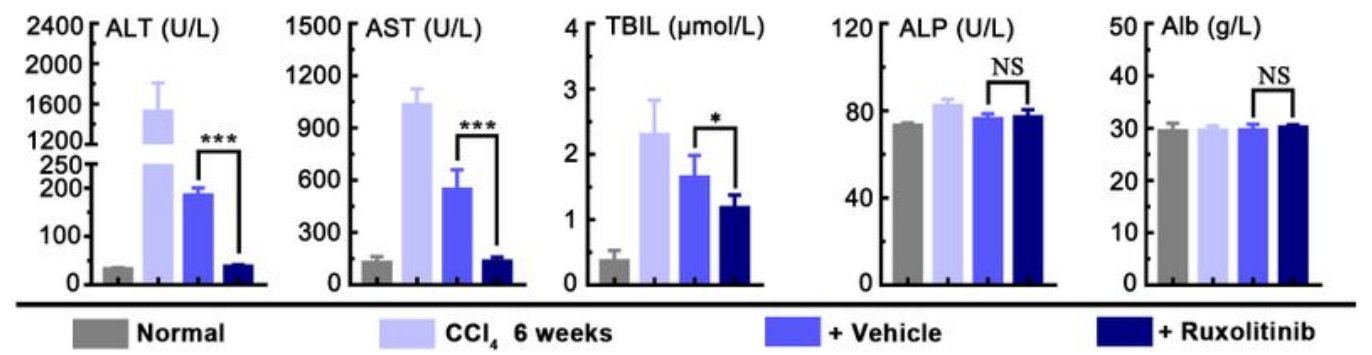

Figure 5

Ruxolitinib accelerates the reversal of liver fibrosis in CCl4 mouse model. (A) Schematic of the experimental design of Ruxolitinib treatment in a CCl4-induced fibrosis reversal model in mice. (B) Representative images of mouse livers stained with H\&E, Sirius red and a-SMA antibodies. (C) Ruxolitinib 
reduced Ishak fibrosis score, and Sirius red, a-SMA IHC staining (magnification 100X). (D) Ruxolitinib reduced the protein expression of p-JAK1, p-JAK2, and a-SMA. (E) Serum levels of ALP, ALT, TBIL, ALP, and Alb. Date presented are means \pm SD. NS = not significant; $*, P<0.05 ; * \star, P<0.01 ; * \star *, P<0.001$.

A

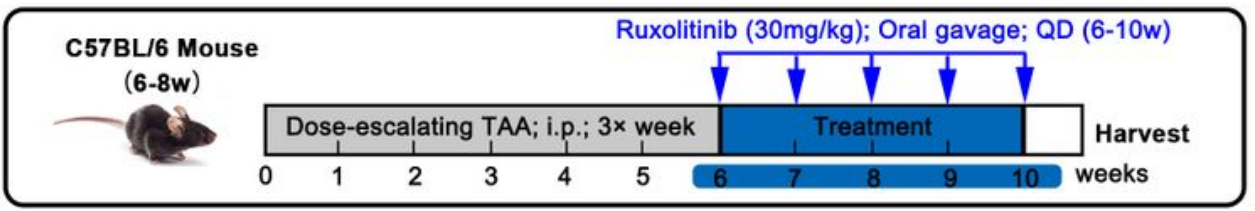

B
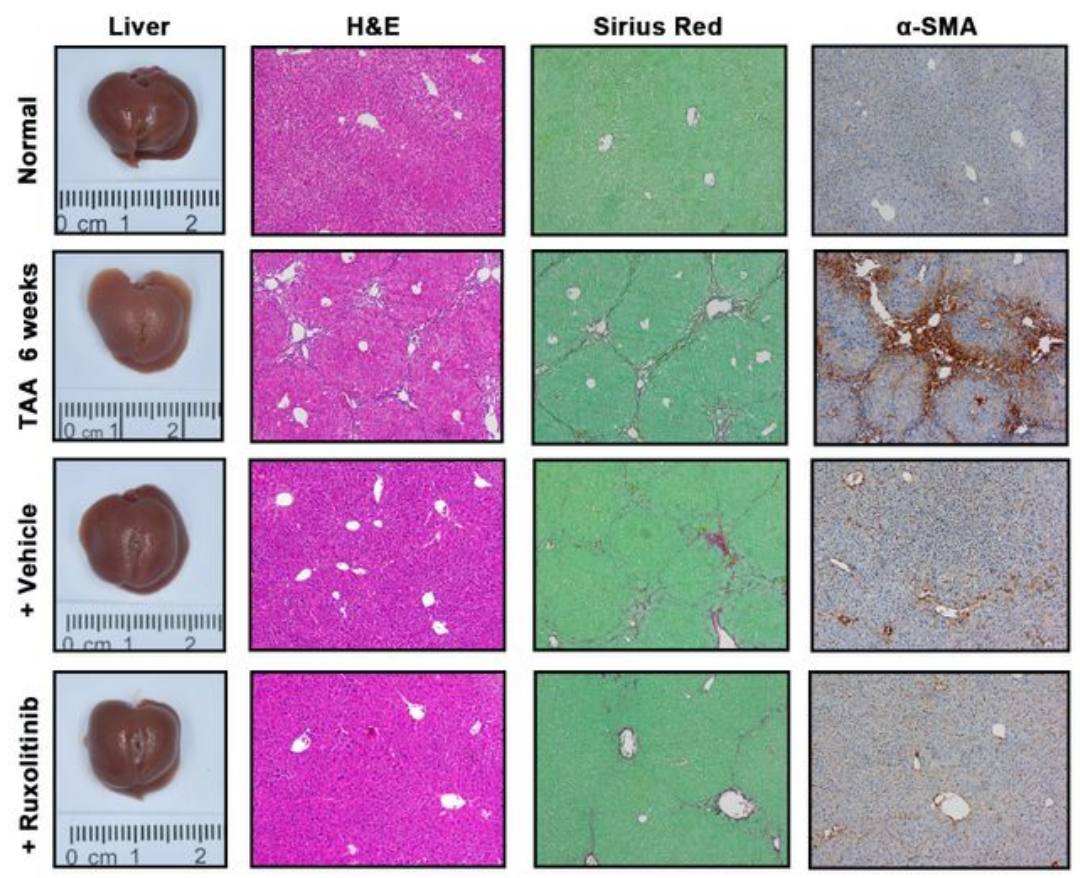

C

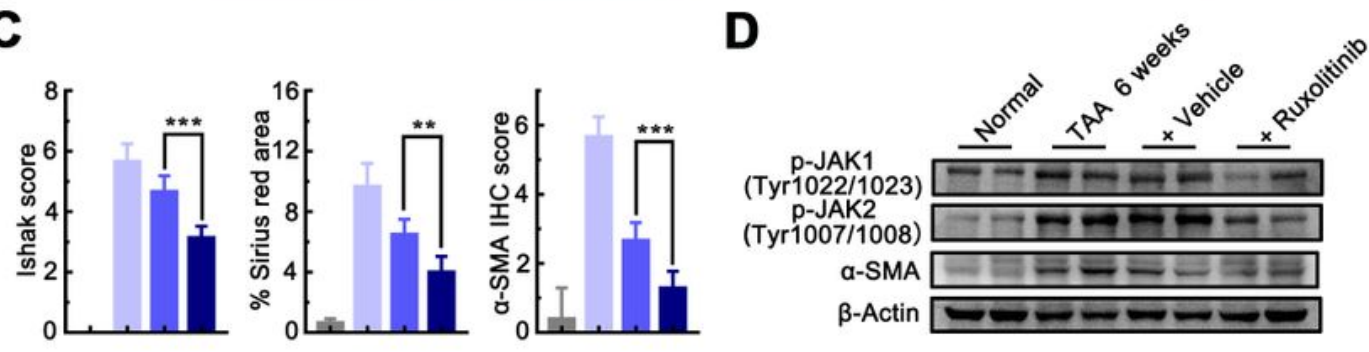

$\mathbf{E}$

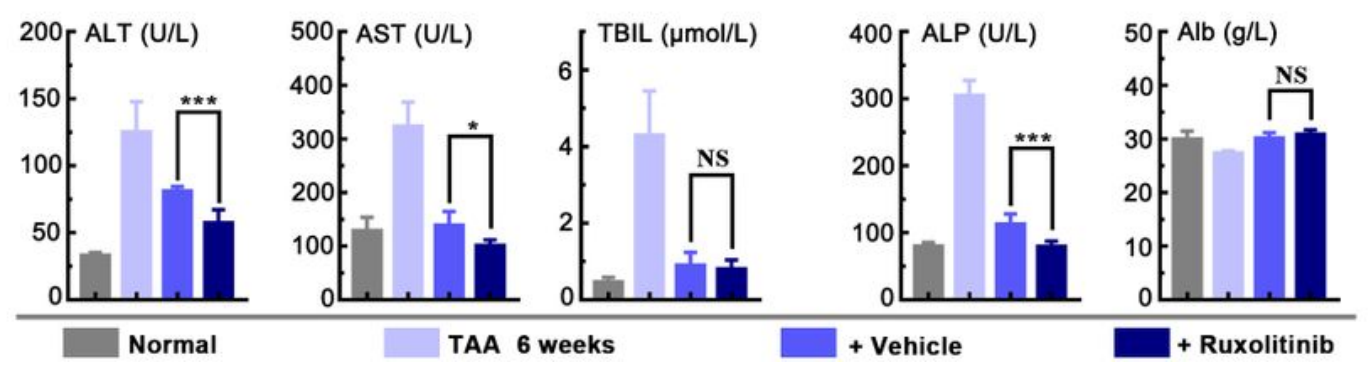

Figure 6

Ruxolitinib accelerates the reversal of liver fibrosis in the TAA mouse model. (A) Schematic of the experimental design of Ruxolitinib treatment in a TAA-induced fibrosis reversal model in mice. (B) 
Representative images of mouse livers stained with H\&E, Sirius red and a-SMA antibodies. (C) Ruxolitinib reduced Ishak fibrosis score, and Sirius red, a-SMA IHC staining (magnification 100X). (D) Ruxolitinib reduced the protein expression of p-JAK1, p-JAK2, and a-SMA. (E) Serum levels of ALP, ALT, TBIL, ALP and Alb. Data presented are means \pm SD. NS = not significant; $*, P<0.05 ; * \star, P<0.01 ; * \star \star, P<0.001$.
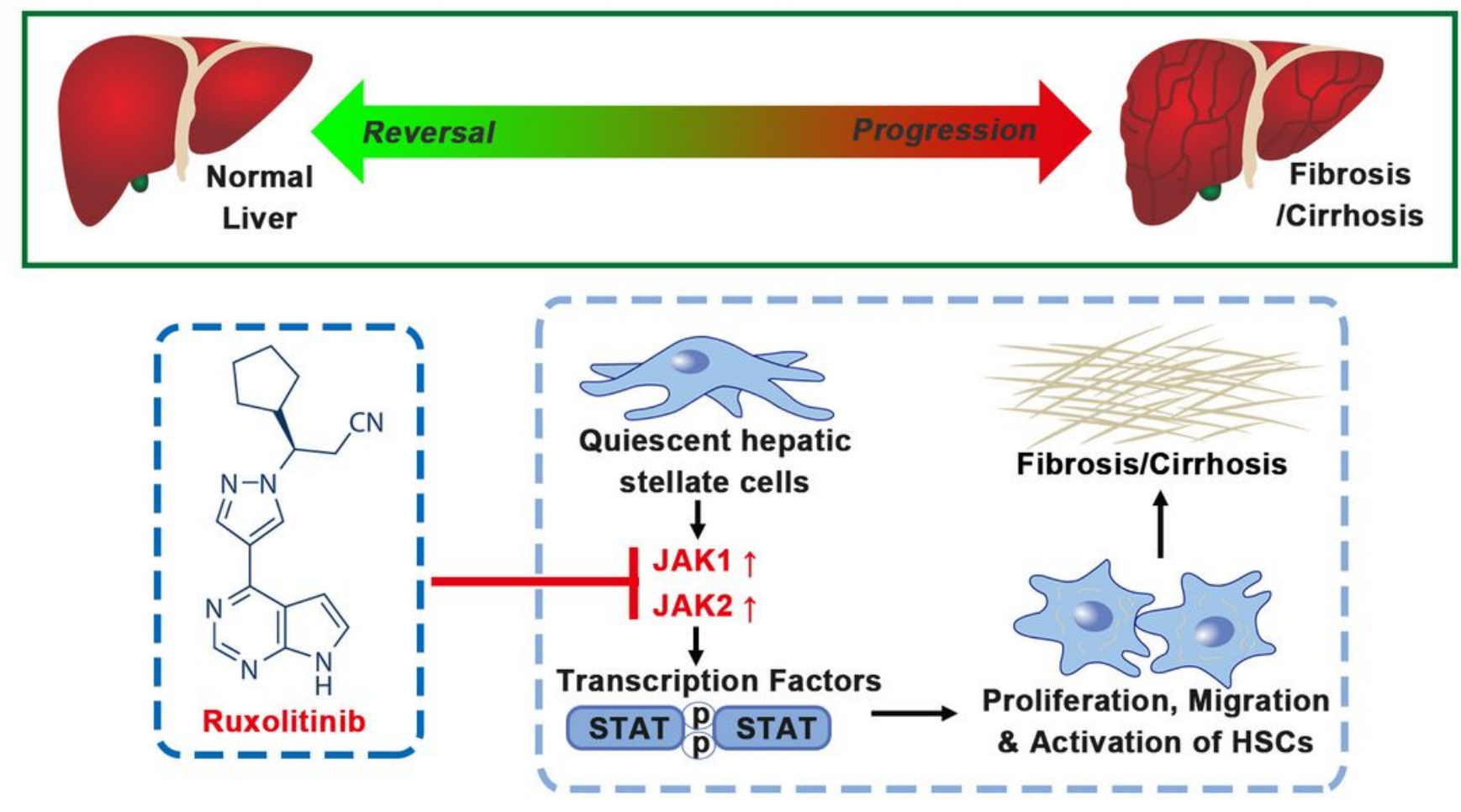

\section{Figure 7}

Proposed model of Ruxolitinib suppresses liver fibrosis progression and accelerates fibrosis reversal via selectively targeting JAK $1 / 2$.

\section{Supplementary Files}

This is a list of supplementary files associated with this preprint. Click to download.

- SupplementalMaterial.pdf 